\title{
The Mitochondrial Fusion-Related Proteins Mfn2 and OPA1 are Transcriptionally Induced during Differentiation of Bone Marrow Progenitors to Immature Dendritic Cells
}

\author{
Seung-Wook Ryu ${ }^{1,2,4,}$, Eun Chun Han ${ }^{3,4}$, Jonghee Yoon', and Chulhee Choi ${ }^{1,2,3, *}$
}

\begin{abstract}
The shape and activity of mitochondria are tightly regulated by fusion and fission processes that are essential for maintaining normal cellular function. However, little is known about the involvement of mitochondrial dynamics in the development of the immune system. In this study, we demonstrate that mitochondrial dynamics play a role in the differentiation and migration of immature dendritic cells (imDCs). We show that mitochondrial elongation is induced during GM-CSF-stimulated differentiation of bone marrow progenitors to imDCs accompanied by upregulation of mitochondrial fusion proteins. These processes precede the changes in mitochondrial morphology and connectivity that occur during differentiation. Mfn2 and OPA1, but not Mfn1, are transcriptionally upregulated during differentiation; however, knockdown of Mfn2 and OPA1 does not induce any change in expression of CD11c, CDC80, or CD86. Notably, knockdown of Mfn2 or OPA1 by siRNA in imDCs significantly reduces CCR7 expression and CCL19-mediated migration. These results suggest that the mitochondrial fusion-related proteins Mfn2 and OPA1 are upregulated during bone marrow progenitor differentiation and promote the migration of imDCs by regulating the expression of CCR7.
\end{abstract}

\section{INTRODUCTION}

Dendritic cells (DCs) are antigen-presenting cells that play a critical role in the adaptive immune response. Bone marrow (BM) progenitors differentiate into immature DCs (imDCs). These imDCs have the ability to take up antigen and are found in the $T$ cell-rich regions of most lymphoid organs, and in many other

\footnotetext{
${ }^{1}$ Department of Bio and Brain Engineering, ${ }^{2} \mathrm{KI}$ for the Biocentury, ${ }^{3}$ Graduate School of Medical Science and Engineering, Korea Advanced Institute of Science and Technology, Daejeon 305-701, Korea, ${ }^{4}$ These authors contributed equally to this work.

*Correspondence: ryus@ kaist.ac.kr (SWR); cchoi@kaist.ac.kr (CC)
}

Received 27 October, 2014; accepted 4 November, 2014; published online 12 November, 2014

Keywords: differentiation, immature dendritic cells, Mfn2, migration, mitochondrial dynamics, OPA1 tissues and organs of the body (Banchereau and Steinman 1998). Mature DCs migrate via lymph vessels to regional lymph nodes (LNs), where they interact with naive $T$ cells to generate either antigen-specific tolerance or immunity. The cellular events of BM progenitor differentiation are well defined by key morphological features and by the presence or absence of various molecules on the cell surface; however, the role of mitochondrial dynamics in BM progenitor differentiation remains unclear.

Mitochondria play important roles in multiple cellular functions, including ATP generation, reactive oxygen species (ROS) production, calcium buffering, cell proliferation and migration, and apoptosis (Aihara et al., 2009; Favre et al., 2010; Ryu et al., 2013; Thundathil et al., 2005). Mitochondrial defects have been implicated in a broad range of metabolic, neoplastic, degenerative, and age-related diseases (Detmer and Chan, 2007; Zhao et al., 2013). Mutation of either mitochondrial DNA (mtDNA) or the nuclear DNA-encoding mitochondrial proteins has been demonstrated to be responsible for many mitochondria-associated pathologies (Vafai and Mootha, 2012). Recent studies show that mitochondria are highly dynamic organelles that undergo continuous fusion and fission to maintain functional integrity (Detmer and Chan, 2007; Liesa et al., 2009). Typically, fusion of the mitochondrial membrane is initiated by outer mitochondrial membrane proteins called mitofusins (Mfn1 and Mfn2). Fusion of the inner mitochondrial membrane is mediated by Optic Atrophy 1 (OPA1) (Santel and Fuller, 2001; Wong et al., 2000). Key molecules involved with the fission process include dynamin 1-like protein (Drp1), mitochondrial fission factor (Mff), and mitochondrial fission 1 protein (Fis1) (Otera et al., 2010). It is now clear that normal mitochondrial morphology is maintained by the balanced action of these nuclear DNA-encoded factors.

Studies have demonstrated the relevance of mitochondrial dynamics for mitochondrial activity and metabolism (Bach et al. 2003; Pich et al., 2005). Mitochondrial biogenesis, featuring an increase in mitochondrial mass/volume, mtDNA copy number, and mitochondrial activity, is induced during myoblast differentiation to enhance cellular metabolic function (Barbieri et al., 2011; Kraft et al., 2006). Typically, the expression of biochemical markers of mitochondrial biogenesis, such as PPAR $\gamma$ coactivator-1 (PGC-1), nuclear respiratory factor-1 $\alpha$ (NRF-1 $\alpha$ ), and mitochondrial transcription factor $\mathrm{A}$ (Tfam), are significantly increased during myogenesis (Barbieri et al., 2011). A recent study reported that proteins of the PGC-1 family, especially 
PGC-1 $\beta$, directly increase the transcriptional level of Mfn2 and induce mitochondrial fusion in muscles (Liesa et al., 2008; Zorzano et al., 2010). Even though one may reasonably speculate that mitochondrial biogenesis is essential for the mitochondrial dynamics required during cell differentiation, the relationship between mitochondrial dynamics and mitochondrial biogenesis in relation to cellular physiology remains poorly understood. In the present study, we demonstrated that Mfn2 and OPA1 mRNA expression is markedly upregulated during mouse bone marrow-derived dendritic cell (BMDC) differentiation. We also observed mitochondrial morphological dynamics during DC differentiation, and showed that changes in the expression of proteins related to mitochondrial dynamics play an important role in the migration of immature DCs.

\section{MATERIALS AND METHODS}

Animals and reagents

Six-week-old female C57BL/6 mice were purchased from Orient Bio (Korea). All mice were maintained in the animal care facility of KAIST according to the KAIST animal care and use guidelines (KA2013-09). Murine granulocyte-macrophage colony-stimulating factor (mGM-CSF) was acquired from R\&D Systems (USA). Lipopolysaccharide (LPS; O111:B4) was obtained from Sigma-Aldrich (USA). FITC-conjugated anti-mouse MHCII, PE-labeled anti-mouse CD80, and CD86 antibodies were purchased from eBioscience (USA). Polyclonal anti-Mfn1 and antiMfn2 antibodies were kindly provided by Dr. Richard J. Youle. Antibodies for cytochrome $c$, OPA1, Drp1, Tom20, APClabeled CD11c, and CD16/CD32 were obtained from BD Biosciences (USA).

\section{Bone marrow-derived dendritic cells}

BMDCs were generated from BM progenitor cells. BM progenitor cells were collected from the femurs and tibiae of 6-weekold female C57BL/6 mice, and treated with ACK lysing buffer (Life Technologies, USA) to remove erythrocytes. Cells were washed and cultured in complete RPMl-1640 media containing $10 \%$ fetal bovine serum (FBS), $100 \mathrm{U} / \mathrm{ml}$ penicillin, $100 \mu \mathrm{g} / \mathrm{ml}$ streptomycin, and $10 \mathrm{ng} / \mathrm{ml} \mathrm{mGM}-\mathrm{CSF}$. After 2 days, the medium was replaced with fresh complete medium containing mGM-CSF. On day 4, fresh complete medium with mGM-CSF was added to the culture in a 1:1 ratio. On days 6 and 7, cells were collected as a source of imDCs. Isolated imDCs were matured by exposure to LPS (100 ng/ml) for $24 \mathrm{~h}$.

\section{Flow cytometry analysis}

For phenotypic analysis of DCs, immunofluorescent labeling was performed. Cells were labeled in FACS buffer at $4^{\circ} \mathrm{C}$ for $20 \mathrm{~min}$ with the following antibodies: FITC-labeled anti-mouse MHCII, PE-labeled anti-mouse CD11c, PE-/FITC-labeled isotype control antibodies, anti-mouse CD80, and anti-mouse CD86. After washing, cells were assayed using a FACSCalibur instrument (BD Biosciences) and CellQuest software.

\section{RNA interference and transfection}

Small double-stranded RNAs (siRNAs) for mouse Drp1, Mfn1, Mfn2, OPA1, and control sequence were purchased from Santa Cruz Biotechnology (USA). BM progenitor cells were isolated and cultured. After 2 days, $1 \times 10^{6}$ cells were reseeded into a 6 well plate. One day after seeding, cells were co-transfected with siRNA and the pEGFP-plasmid using Lipofectamine RNAiMAX (Invitrogen, USA). After 1 day of transfection, the medium was refreshed and the cells were grown for a further 2 days. For analysis of the effects on DC maturation, $1 \times 10^{6}$ immature DCs were reseeded and co-transfected with siRNA and the pEGFPplasmid using Lipofectamin RNAiMAX. After 1 day, the medium was replaced with fresh complete RPMl with $10 \mathrm{ng} / \mathrm{ml}$ LPS, and the cells were grown for a further 1 day.

\section{Analysis of gene expression}

Total RNA was extracted from BMDCs according to the manufacturer's instructions (Qiagen, USA). Then 0.5-1 $\mu \mathrm{g}$ of total RNA was used for each RT-PCR assay (Applied Biosystems, USA). Quantification of mRNA was performed using StepOne Real-Time PCR (Applied Biosystems). Expression levels were normalized against an endogenous control (GAPDH). The following gene-specific primers were used for quantitative PCR: mouse Drp1 sense 5'-TTAGTGGCAATTGAGCTAGCGTAT-3' and antisense 5'-CCCACAGGCATCAGCAAAGT-3'; mouse Mfn1 sense 5'-GACGACCCGTGCGAAAGA-3' and antisense 5'-AGCTTCTCGGTTGCATAGGGGACA-3'; mouse Mfn2 sense 5'-CGAGGCTCTGGATTCACTTCA-3' and antisense 5'-CCAACCAGCCAGCTTTATTCC-3'; mouse OPA1 sense 5'-CCTTTGTCGCAGAGGTTTTTATTAC-3' and antisense 5'-CATTGCATTCAGCTCAGAATC-3'; mouse GAPDH sense 5'-TGGCCTCCAGATAAGAAAC-3' and antisense 5'-GGGATAGGGCCCTCTCT- 3 '. The data were quantified using the comparative threshold cycle $(\mathrm{Ct})$ method for relative gene expression.

\section{Immunofluorescence}

Cells grown in 6-well plates were fixed by incubation with $4 \%$ paraformaldehyde for $15 \mathrm{~min}$ at room temperature, permeabilized, and then blocked by incubation with 3\% bovine serum albumin in PBS for $45 \mathrm{~min}$ at room temperature. Cells were incubated with the primary antibodies indicated in the figures. After washing with PBS, cells were incubated with Alexa Fluor 488-conjugated goat anti-mouse IgG or goat anti-rabbit IgG as the secondary antibody. Cells were observed under a Zeiss LSM 510 confocal microscope. The lengths of single tubular mitochondria were analyzed using LSM Image Browser software (Carl Zeiss AG). Data represent the means \pm standard deviation (SD) of experiments, each with 10 cells per condition.

\section{Fluorescence recovery after photobleaching}

BMDCs were incubated with MitoTracker Orange CMTMRos (Invitrogen) for $5 \mathrm{~min}$, and then washed with fresh RPMI-1640. Live cells were imaged using a Zeiss LSM 510. For fluorescence recovery after photobleaching (FRAP) analysis, the MitoTracker fluorescence in a small region of interest (ROI) was photobleached until the fluorescence signal of the region was abolished. The region was then monitored for fluorescence recovery. The fluorescence intensity was normalized against the intensity of the $\mathrm{ROI}$ in the first image of the series, and fluorescence intensity recovery rates were plotted.

\section{Chemotactic cell migration}

Cell migration assays were performed using $5.0-\mu \mathrm{m}$ pore size Transwell permeable supports (Corning Costar, USA). The lower chamber was filled with $300 \mathrm{ng} / \mathrm{ml} \mathrm{CCL19}$. Then $1.5 \times 10^{5}$ BMDCs were added to the upper chamber and then incubated for $4 \mathrm{~h}$ at $37^{\circ} \mathrm{C}$. Cells that migrated to the lower chamber were collected and counted by flow cytometry for a fixed time period of 60 s using CellQuest software.

\section{Statistical analysis}

All experiments were repeated at least three times with consistent results. Statistical data are presented as the mean \pm 
A

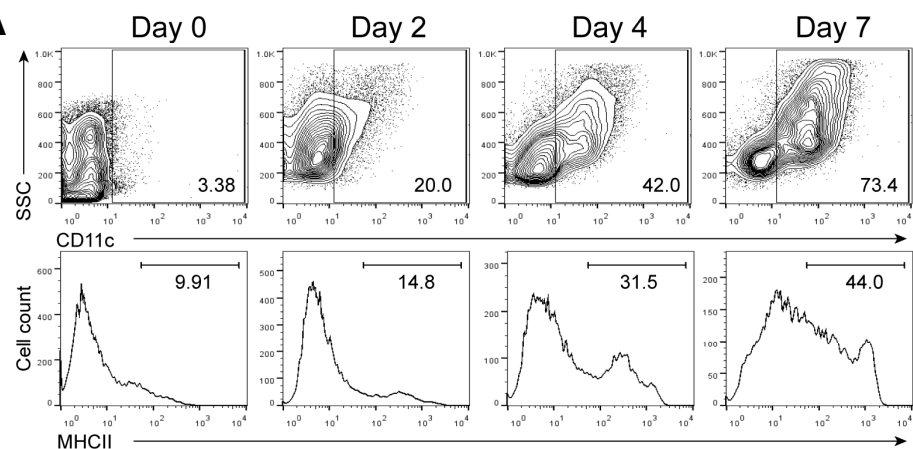

B

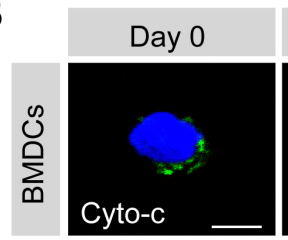

Day 2

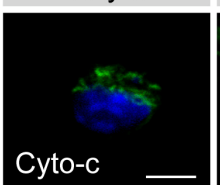

Day 4

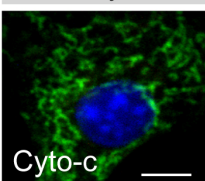

Day 7

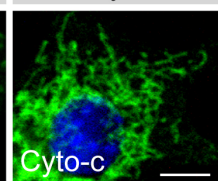

C

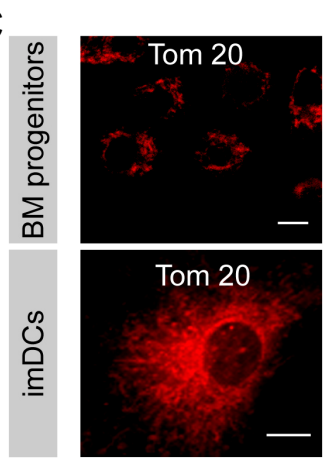

E

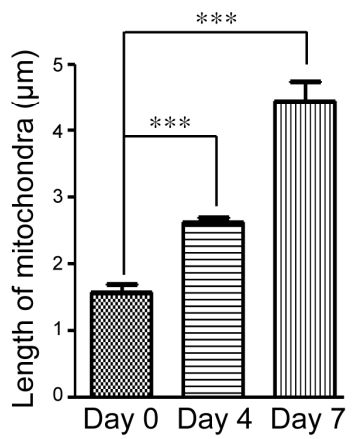

D

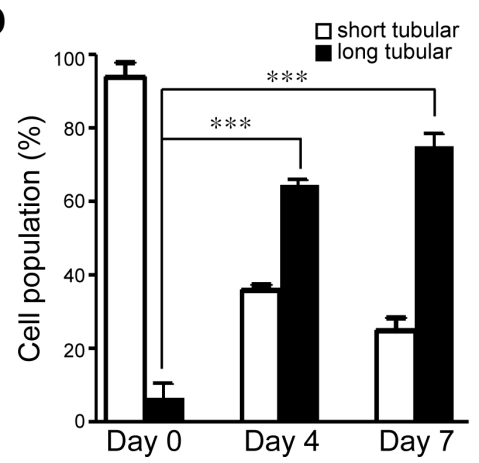

\section{$\boldsymbol{F}$}

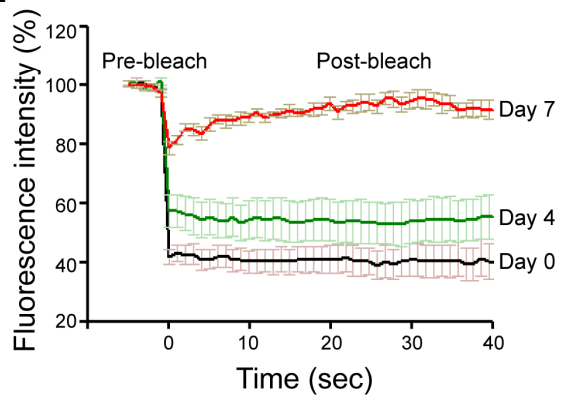

Fig. 1. Mitochondrial morphology during mouse BM progenitor differentiation. (A) Change in cell surface molecule expression during differentiation. BM progenitors were treated with mGM-CSF for 7 days. The expression of cell surface molecules CD11c (upper panel) and $\mathrm{MHCll}$ (bottom panel) were assessed by flow cytometry. (B-E) Change in mitochondrial morphology during differentiation. Cells were fixed and labeled with DAPI [nuclei; blue; (B)], APC-labeled CD11c (B), cytochrome $c$ [Cyto-C; green; (B)], or Tom 20 [mitochondria; red; $(C)$ ]. Mitochondrial morphology was quantified and plotted (D). Mitochondrial length was analyzed and plotted (E). imDCs, immature dendritic cells. Data represent the mean \pm SD of three experiments. ${ }^{* \star *} p<0.0005$. Bar, $5 \mu \mathrm{m}$. (F) Enhancement of mitochondrial fusion activity during differentiation. Cells were cultured with mGM-CSF for the indicated days. Live cells were stained with Mitotracker CMTMRos and then photobleached. The photobleached region of interest $(\mathrm{ROI})$ was monitored for fluorescence recovery. Each line represents the mean of more than 20 measurements. standard deviation (SD). Levels of significance for comparisons between two independent samples were determined using Student's $t$-test. Comparisons of group means were assessed by analysis of variance (ANOVA) with Tukey's honestly significant post hoc test being applied to significant main effects. A pvalue of $<0.05$ was considered statistically significant.

\section{RESULTS AND DISCUSSION}

Changes in mitochondrial dynamics during GM-CSFinduced differentiation of bone marrow progenitors

To investigate the regulation of mitochondrial dynamics during BM progenitor differentiation, BM progenitors were collected from the femurs and tibiae of C57BL/6 mice and cultured in the presence of mGM-CSF for 7 days. Flow cytometric analysis of the imDC surface marker proteins $\mathrm{CD} 11 \mathrm{c}$ and $\mathrm{MHCll}$ demonstrated that this treatment caused the majority of BM progenitors to differentiate into imDCs (Supplementary Fig. 1A). To verify the differentiation of $\mathrm{BM}$ progenitors into imDCs, we evaluated the change in expression of cell surface molecules involved with antigen presentation following LPS-induced imDC maturation. Flow cytometric analysis revealed that cell surface expression of CD86 and CD80 was significantly upregulated by LPS treatment (Supplementary Fig. 1B). Tumor necrosis factor (TNF)- $\alpha$ and interleukin (IL)-12, which are involved in the induction of the T-cell response, were also highly upregulated in 
$\boldsymbol{A}$

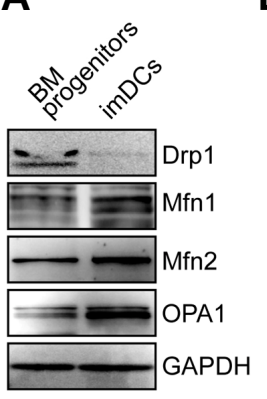

$B$

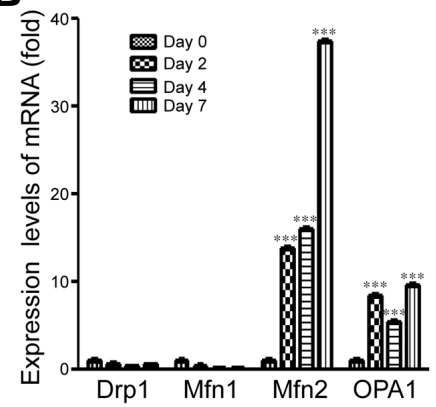

C

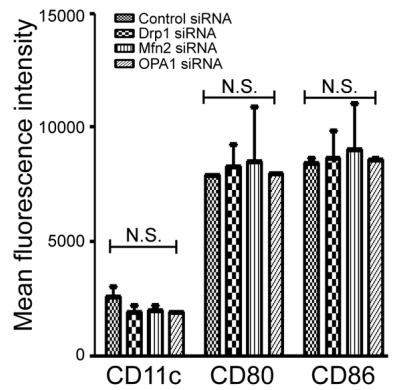

Fig. 2. The mitochondrial fusion-related factors Mfn2 and OPA1 are transcriptionally induced during differentiation. (A) Expression of mitochondrial fusion-related proteins Mfn2 and OPA1. BM progenitors, or imDCs cultured in the presence of mGM-CSF for 7 days, were harvested and subjected to Western blotting with the indicated antibodies. GAPDH was used as a loading control. (B) mRNA level of Mfn2 and OPA1 during differentiation. The mRNA level was assessed by quantitative real-time PCR using the indicated primers.

Expression levels were normalized against an endogenous control: mouse GAPDH. (C) Effects of mouse Mfn2 and OPA1 knockdown on the imDC phenotype. BM progenitors were co-transfected with the pEGFP plasmid and the indicated siRNA, and then maintained for a further 4 days. GFP-positive cells were analyzed with anti-CD11c, CD80, and CD86 antibodies using a FACSCalibur flow cytometer. N.S., not significant. Data represent the mean $\pm \mathrm{SD}$ of three experiments. ${ }^{* \star} p<0.0005$.

LPS-matured DCs (Supplementary Fig. 1C).

To evaluate the correlation between mitochondrial morphology and BM progenitor differentiation, the expression levels of the surface marker proteins CD11c and MHCIl were analyzed at various time points. Less than $20 \%$ of the BM progenitors were differentiated on day 2 , whereas most $(73.3 \pm 3 \%)$ of the cells had differentiated to imDCs on day 7 (Fig. 1A). The mitochondrial tubular network in the imDCs at day 7 was more highly interconnected compared to that of the DC progenitors at day 0 , as assessed by immunolabeling of the mitochondrial proteins cytochrome $c$ (inter membrane space protein; Fig. 1B) and Tom 20 (outer membrane protein; Fig. 1C). The mitochondria of BM progenitors were frequently located near the nucleus (on day 0 and day 2; Fig. 1B). Most (93 $\pm 4 \%$ ) of the BM progenitors contained mitochondria with a short, tubular morphology (1.5 \pm 0.1 $\mu \mathrm{m}$; Figs. 1D and 1E). Moreover, most (60-70\%) of the cells on days 4-7 showed an increased quantity of mitochondria that were typically long and tubular in shape (2.6-4.4 $\pm 0.3 \mu \mathrm{m}$; Figs. 1B-1E) and distributed diffusely throughout the cytosol, suggesting that mitochondrial fusion activity is induced during differentiation. To determine whether mitochondrial fusion activity is required for the formation and distribution of elongated mitochondria during differentiation of BM progenitors, we quantitatively analyzed the mitochondrial connectivity in live cells using a fluorescence recovery after photobleaching (FRAP) technique. In BM progenitors (day 0; Fig. 1F), the fluorescence in the photo-bleached area did not recover. In contrast, the photobleached fluorescence recovered slowly in cells at day 4 of differentiation, and rapidly in cells at day 7 (Fig. 1F). These results clearly confirm that mitochondrial fusion dynamics are highly induced in the late stages of differentiation of BM progenitors to imDCs. In recent studies, rotenone, a mitochondrial complex I inhibitor, caused inhibition of human DC differentiation via inhibition of ROS generation (Del Prete et al., 2008). An increase in mitochondrial number and metabolism is manifested by DC differentiation (Zaccagnino et al., 2012). Similarly, depletion of mitochondrial phosphatase Ptpmt 1 from embryonic stem (ES) cells decreased aerobic metabolism and blocked differentiation (Shen et al., 2011). Thus, we could propose that mitochondrial activity including mitochondrial fusion is strongly involved during cell differentiation.

\section{Mfn2 and OPA1 are transcriptionally induced during} differentiation of BM progenitors to imDCs

Next, we investigated whether mitochondrial fusion activity during the differentiation of BM progenitors is associated with changes in factors involved with mitochondrial dynamics. Ex- pression levels of mitochondrial fusion proteins, including Mfn1, Mfn2, and OPA1, increased during differentiation of BM progenitors, whereas the levels of Drp1 decreased (Fig. 2A and Supplementary Fig. 1D). To investigate whether expression of mitochondrial fusion proteins is transcriptionally regulated in imDCs, we analyzed mRNA expression using quantitative RTPCR. Consistent with the Western blot analysis, mRNA levels of Mfn2 and OPA1, but not Mfn1, were dramatically induced in a time-dependent manner (Fig. 2B). Moreover, mRNA expression of Mfn2 and OPA1 was induced 13-fold and 8-fold, respectively, at an early stage of BM progenitor differentiation (day 2). Their expression levels were highest on day 7 (Mfn2: 37-fold, OPA1: 9-fold), when the BM progenitors were fully differentiated into imDCs. These results clearly indicate that mitochondrial elongation during differentiation is related to transcriptional upregula-tion of Mfn2 and OPA1. Consistent with these data, the appearance of enlarged mitochondria in connection with Mfn2 and OPA1 upregulation was reported in erythropoietic cell differentiation during copper deficiency (Bustos et al., 2013; Dallman and Goodman, 1970). Wasilewski et al. (2012) showed that steroidogenesis of trophoblasts was accompanied by mitochondrial fission and a reduction of Mfn2 and OPA1 expression. Zaccagnino et al. (2012) also reported that variables associated with mitochondrial biogenesis, including mitochondrial mass and mtDNA copy number, are increased by the activity of transcription factors including the PGC-1 group, Tfam and Nrf-1, during cellular differentiation. A recent study demonstrated that the PGC-1 family (especially PGC-1 $1 \beta$ ) directly increased the transcriptional level of Mfn2 and induced mitochondrial fusion during myoblast differentiation (Liesa et al., 2008; Zorzano et al., 2010). Mitochondrial biogenesis is diminished during myoblast differentiation in OPA1 heterozygous null mice (Caffin et al., 2013). In the present study, we confirmed that mitochondrial elongation during differentiation is associated with upregulation of Mfn2 and OPA1 expression, suggesting that transcriptional upregulation of Mfn2 and OPA1 is coupled to cellular differentiation.

\section{Knockdown of Mfn2 and OPA1 did not affect the cell surface phenotype of imDCs}

To confirm the functional effect of Mfn2 and OPA1 during DC differentiation, we evaluated the expression of surface marker molecules on imDCs after siRNAs knockdown of proteins with roles related to mitochondrial dynamics. To mitigate the problem of lower efficiency of transfection in BM progenitors, cells on day 3 of differentiation were transiently co-transfected with the 
target siRNA together with the pEGFP-plasmid and then analyzed by flow cytometry after a further 3 days. Flow cytometric analysis demonstrated that the majority of the EGFP-positive cells transfected with control siRNA expressed the cell surface molecules CD11c, CD80, and CD86 (Fig. 2C). The expression of $\mathrm{CD11c}$ was slightly reduced in the cells transfected with siRNA for Mfn2 or OPA1, but the difference was not statistically significant. In addition, the knockdown of Mfn2 or OPA1 did not induce any change in CD80 and CD86 expression. For functional analysis of imDCs, we assessed the levels of co-stimulatory molecules (CD80 and CD86) during LPS-mediated maturation of DCs. Flow cytometric analysis indicated that the level of expression of Mfn2 and OPA1 did not influence LPS-induced imDC activation (Supplementary Fig. 2). These data collectively indicate that the change in mitochondrial dynamics may not be linked to the expression of cell surface molecules on imDCs and mature DCs.

Knockdown of Mfn2 and OPA1 significantly reduced CCR7 expression, and inhibited CCL19-mediated imDC migration To test whether Mfn1 and OPA1 are functionally relevant to imDCs, cells at day 7 of mGM-CSF-induced differentiation were transiently transfected with target siRNAs. The target mRNA was effectively silenced by $40-50 \%$ in imDCs compared to cells with control siRNA. Knockdown of mitochondrial fusion-related Mfn2 or OPA1 significantly reduced the expression of CCR7 (Fig. 3A). In contrast, knockdown of mitochondrial fission-related Drp1 had no effect on CCR7 expression (Fig. 3A). To confirm the effect of Mfn2 and OPA1 on CCR7-dependent migration, we utilized Transwells to assay CCL19-stimulated migration (Fig. 3B). Knockdown of Drp1 slightly increased imDC migration, but the difference was not statistically significant. However, consistent with the CCR7 level in imDCs, the chemotactic migration of imDCs toward CCL19 was significantly repressed by knockdown of Mfn2 or OPA1 compared to control siRNA-transfected cells (Fig. 3B). We also demonstrated that CCR7 expression was not upregulated by LPS in control siRNA-transfected cells (data not shown). These results clearly indicate that Mfn2 and OPA1, which are upregulated in imDCs, are directly involved in imDC migration without maturation. In contrast, migration of mature DCs has been shown to be mediated by CCR7 binding to the chemokines CCL19 and CCL21 (Sallusto et al., 2000). Consistent with our results, Geissman and colleagues (Geissmann et al., 2002) reported that DC migration and maturation can be independently regulated events and that DC migration can occur without maturation. Toki et al. (2013) reported that the PGI2 analog cicaprost increased the migration of imDCs through upregulation of CCR7 in vitro and in vivo. Thus, our findings support that migration of imDCs can occur without maturation.

Our previous study showed that blocking mitochondrial fission via OPA3 depletion induced mitochondrial elongation and increased the migration of retinal pigment epithelial cells in response to TGF- $\beta$ treatment (Ryu et al., 2013). In contrast, mitochondrial fusion mediated by Mfn1 and OPA1 prevented the migration of T-lymphocytes toward CXCL12 gradients (Campello et al., 2006). Zhao et al. (2013) reported that mitochondrial fission mediated by Drp1 increased the migration of human breast cancer cells. These results show that modulation of mitochondrial dynamics is closely linked to cell migration in a tissue-specific manner.

In summary, we demonstrated that the mitochondrial fusion machinery, especially Mfn2 and OPA1, is induced during mGM-CSF-mediated differentiation of BM progenitors and is
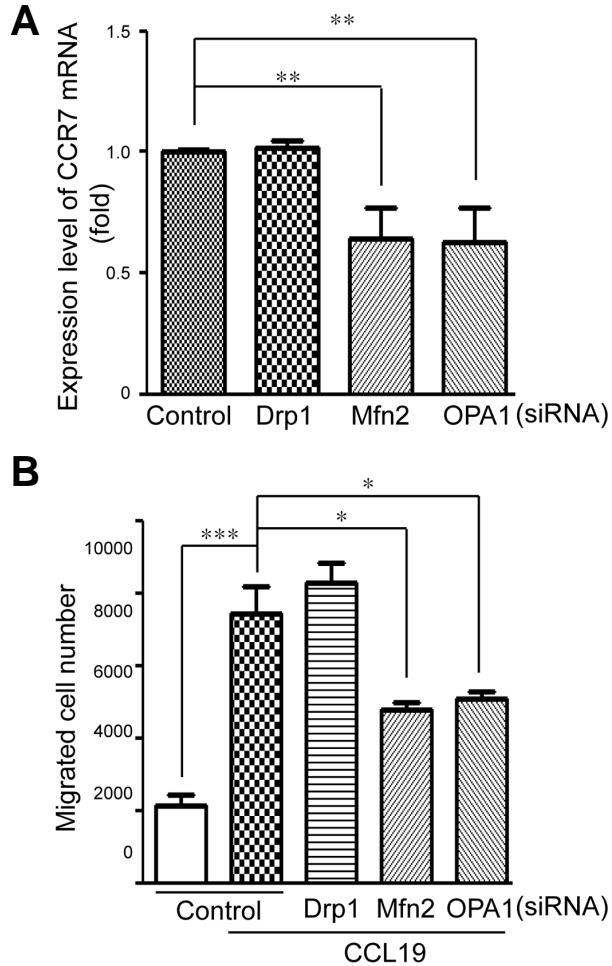

Fig. 3. Knockdown of Mfn2 and OPA1 reduces the migration of imDCs. (A) CCR7 levels in imDCs following knockdown of mouse Mfn2 and OPA1. Cells were transfected with the indicated siRNA. mRNA levels were analyzed by quantitative RT-PCR using a specific CCR7 primer. Expression levels were normalized against an endogenous control: mouse GAPDH. (B) CCL19-mediated migration of imDCs was reduced by knockdown of mouse Mfn2 and OPA1. imDCs transfected with specific siRNA were cultured in a migration chamber. Cells that migrated were counted by flow cytometry. Data represent the mean \pm SD of three experiments. ${ }^{*} p$ $<0.05 ;{ }^{* *} p<0.005 ;{ }^{* * *} p<0.0005$.

essential for the migration of imDCs. A notable finding is that Mfn2 and OPA1, while weakly expressed in BM progenitors, are transcriptionally upregulated during differentiation to imDCs. Furthermore, mitochondria become highly elongated as a result of an increase in mitochondrial fusion activity during the differentiation process. We also demonstrated that Mfn2 and OPA1 are not strongly involved in differentiation, but pay a role in imDC migration by regulating CCR7 expression. These results collectively provide further insight into the molecular mechanism underlying DC differentiation and cellular function, and will facilitate the development of therapeutic strategies involving manipulation of BM progenitors.

Note: Supplementary information is available on the Molecules and Cells website (www.molcells.org).

\section{ACKNOWLEDGMENTS}

This work was supported by the Basic Science Research Program through the National Research Foundation of Korea (NRF) funded by the Ministry of Education, Science and Technology (2012007370), and by the Korean Health Technology R\&D project, the Ministry of Health \& Welfare (A111025), Republic of Korea. 


\section{REFERENCES}

Aihara, T., Nakamura, N., Honda, S., and Hirose, S. (2009). A novel potential role for gametogenetin-binding protein 1 (GGNBP1) in mitochondrial morphogenesis during spermatogenesis in mice. Biol. Reprod. 80, 762-770.

Bach, D., Pich, S., Soriano, F.X., Vega, N., Baumgartner, B., Oriola, J., Daugaard, J.R., Lloberas, J., Camps, M., Zierath, J.R., et al. (2003). Mitofusin-2 determines mitochondrial network architecture and mitochondrial metabolism. A novel regulatory mechanism altered in obesity. J. Biol. Chem. 278, 17190-17197.

Banchereau, J., and Steinman, R.M. (1998). Dendritic cells and the control of immunity. Nature 392, 245-252.

Barbieri, E., Battistelli, M., Casadei, L., Vallorani, L., Piccoli, G. Guescini, M., Gioacchini, A.M., Polidori, E., Zeppa, S., Ceccaroli, P., et al. (2011). Morphofunctional and biochemical approaches for studying mitochondrial changes during myoblasts differentiation J. Aging Res. 2011, 845379.

Bustos, R.I., Jensen, E.L., Ruiz, L.M., Rivera, S., Ruiz, S., Simon, F., Riedel, C., Ferrick, D., and Elorza, A.A. (2013). Copper deficiency alters cell bioenergetics and induces mitochondrial fusion through up-regulation of MFN2 and OPA1 in erythropoietic cells. Biochem. Biophys. Res. Commun. 437, 426-432.

Caffin, F., Prola, A., Piquereau, J., Novotova, M., David, D.J., Garnier A., Fortin, D., Alavi, M.V., Veksler, V., Ventura-Clapier, R., et al. (2013). Altered skeletal muscle mitochondrial biogenesis but improved endurance capacity in trained OPA1-deficient mice. J. Physiol. 591, 6017-6037.

Campello, S., Lacalle, R.A., Bettella, M., Manes, S., Scorrano, L., and Viola, A. (2006). Orchestration of lymphocyte chemotaxis by mitochondrial dynamics. J. Exp. Med. 203, 2879-2886.

Dallman, P.R., and Goodman, J.R. (1970). Enlargement of mitochondrial compartment in iron and copper deficiency. Blood 35, 496-505

Del Prete, A., Zaccagnino, P., Di Paola, M., Saltarella, M., Oliveros Celis, C., Nico, B., Santoro, G., and Lorusso, M. (2008). Role of mitochondria and reactive oxygen species in dendritic cell differentiation and functions. Free Radic. Biol. Med. 44, 14431451.

Detmer, S.A., and Chan, D.C. (2007). Functions and dysfunctions of mitochondrial dynamics. Nat. Rev. Mol. Cell. Biol. 8, 870-879.

Favre, C., Zhdanov, A., Leahy, M., Papkovsky, D., and O'Connor, R. (2010). Mitochondrial pyrimidine nucleotide carrier (PNC1) regulates mitochondrial biogenesis and the invasive phenotype of cancer cells. Oncogene 29, 3964-3976.

Geissmann, F., Dieu-Nosjean, M.C., Dezutter, C., Valladeau, J., Kayal, S., Leborgne, M., Brousse, N., Saeland, S., and Davoust, J. (2002). Accumulation of immature Langerhans cells in human lymph nodes draining chronically inflamed skin. J. Exp. Med. 196, 417-430.

Kraft, C.S., LeMoine, C.M., Lyons, C.N., Michaud, D., Mueller, C.R., and Moyes, C.D. (2006). Control of mitochondrial biogenesis during myogenesis. Am. J. Physiol. Cell Physiol. 290, C11191127.

Liesa, M., Borda-d'Agua, B., Medina-Gomez, G., Lelliott, C.J., Paz, J.C., Rojo, M., Palacin, M., Vidal-Puig, A., and Zorzano, A. (2008) Mitochondrial fusion is increased by the nuclear coactivator PGC1beta. PLoS One 3, e3613.
Liesa, M., Palacin, M., and Zorzano, A. (2009). Mitochondrial dynamics in mammalian health and disease. Physiol. Rev. 89, 799-845.

Otera, H., Wang, C., Cleland, M.M., Setoguchi, K., Yokota, S., Youle, R.J., and Mihara, K. (2010). Mff is an essential factor for mitochondrial recruitment of Drp1 during mitochondrial fission in mammalian cells. J. Cell Biol. 191, 1141-1158.

Pich, S., Bach, D., Briones, P., Liesa, M., Camps, M., Testar, X., Palacin, M., and Zorzano, A. (2005). The Charcot-Marie-Tooth type 2A gene product, Mfn2, up-regulates fuel oxidation through expression of OXPHOS system. Hum. Mol. Genet. 14, 14051415.

Ryu, S.W., Yoon, J., Yim, N., Choi, K., and Choi, C. (2013) Downregulation of OPA3 is responsible for transforming growth factor-beta-induced mitochondrial elongation and F-actin rearrangement in retinal pigment epithelial ARPE-19 cells. PLoS One 8 , e63495.

Sallusto, F., Mackay, C.R., and Lanzavecchia, A. (2000). The role of chemokine receptors in primary, effector, and memory immune responses. Annu. Rev. Immunol. 18, 593-620.

Santel, A., and Fuller, M.T. (2001). Control of mitochondrial morphology by a human mitofusin. J. Cell Sci. 114, 867-874.

Shen, J., Liu, X., Yu, W.M., Liu, J., Nibbelink, M.G., Guo, C., Finkel $\mathrm{T}$, and Qu, C.K (2011). A critical role of mitochondrial phosphatase Ptpmt1 in embryogenesis reveals a mitochondria metabolic stress-induced differentiation checkpoint in embryonic stem cells. Mol. Cell Biol. 31, 4902-4916.

Thundathil, J., Filion, F., and Smith, L.C. (2005). Molecular contro of mitochondrial function in preimplantation mouse embryos. Mol. Reprod. Dev. 71, 405-413.

Toki, S., Goleniewska, K., Huckabee, M.M., Zhou, W., Newcomb, D.C., Fitzgerald, G.A., Lawson, W.E., and Peebles, R.S., Jr. (2013). PGI(2) signaling inhibits antigen uptake and increases migration of immature dendritic cells. J. Leukoc. Biol. 94, 77-88.

Vafai, S.B., and Mootha, V.K. (2012). Mitochondrial disorders as windows into an ancient organelle. Nature 491, 374-383.

Wasilewski, M., Semenzato, M., Rafelski, S.M., Robbins, J., Bakardjiev, A.I., and Scorrano, L. (2012). Optic atrophy 1dependent mitochondrial remodeling controls steroidogenesis in trophoblasts. Curr. Biol. 22, 1228-1234.

Wong, E.D., Wagner, J.A., Gorsich, S.W., McCaffery, J.M., Shaw, J.M., and Nunnari, J. (2000). The dynamin-related GTPase, $\mathrm{Mgm1} 1 \mathrm{p}$, is an intermembrane space protein required for maintenance of fusion competent mitochondria. J. Cell Biol. 151, 341-352.

Zaccagnino, P., Saltarella, M., Maiorano, S., Gaballo, A., Santoro, G., Nico, B., Lorusso, M., and Del Prete, A. (2012). An active mitochondrial biogenesis occurs during dendritic cell differentiation. Int. J. Biochem. Cell Biol. 44, 1962-1969.

Zhao, J., Zhang, J., Yu, M., Xie, Y., Huang, Y., Wolff, D.W., Abel, P.W., and Tu, Y. (2013). Mitochondrial dynamics regulates migration and invasion of breast cancer cells. Oncogene 32, 4814-4824.

Zorzano, A., Hernandez-Alvarez, M.I., Palacin, M., and Mingrone, G. (2010). Alterations in the mitochondrial regulatory pathways constituted by the nuclear co-factors PGC-1alpha or PGC-1beta and mitofusin 2 in skeletal muscle in type 2 diabetes. Biochim. Biophys. Acta 1797, 1028-1033. 